\section{Of faster brains and bigger teeth}

SIR-According to Calvin" "slowed human development" is naturally accompanied by slow-growing teeth. It is a common fallacy, however, that the modern human growth period is "slowed". What characterizes modern humans as unique is a prolongation of the postnatal growth period and not a generalized shift in the rate of growth ${ }^{2-5}$. Large human brains are energetically expensive to grow ${ }^{t}$ but this calorific expense is offset by prolonged inhibition of somatic growth ${ }^{3.7}$ until brain growth is completed. Early hominids 'fueled' enlarging brains either with a reduction in body size, or by prolonging the period of brain growth and delaying somatic growth to later times.

Independent of this, local mechanisms exist whereby teeth of all sizes can grow either quickly or slowly within the constraints of the times available to each tooth during the growth period ${ }^{8-10}$. Beynon and Wood ${ }^{11}$ show clearly that natural selection favouring large posterior teeth in Paranthropus has operated directly upon these local mechanisms. Their fast-growing teeth, then, are not the result of a "long indirect path that confounds straightforward adaptationist reasoning", as Calvin would have it.

M. Christopher Dean

Department of Anatomy and

Developmental Biology,

University College and Middlesex

School of Medicine,

Gower Street,

London WCIE 6BT, UK

1. Calvin. W.H. Nature 328, 481 (1987)

2. Lovtrup. S. Syst. Zool. 27, 125-130(1978)

3. Watts. E.S. in Nonhuman Primate Models for Human Growth and Development (ed. Watts. E.S.) 41-65 (Liss New York. 1985)

4. Gould, S. Ontogeny and Phylogeny (Harvard University Press, Cambridge, Massachusetts. 1977)

5. Dean. M.C. \& Wood. B.A. Folia Primat. 43, 157-180 (1984).

6. Martin. R.D. 52nd James Arthur Lecfure (Am. Mus. Nat. Hist... New York, 1983)

. Williams. R.F. \& Hogden. G.D. Am. J. Primat. Suppl. 1 $181(1982)$

8. Dean, M.C. \& Wood. B.A. Folia Primat. 36, 111-127 (1981)

9. Shellis, R.P. Archs oral Biol. 29, 697 - 705 (1984).

10. Dean M.C Am. J phy's. Anthrop. 68, $233-238(1985)$

11. Beynon. A.D. \& Wood, B.A. Nature 326, $493-496$ (1987)

\section{Low levels of aluminium in tea}

SIR-Considerable public concern $^{1-3}$ about the undesirable effects of dietary aluminium has in particular focused on the supposedly high levels of aluminium in tea, reputed ${ }^{4}$ to be $40-100 \mathrm{mg} \mathrm{l}^{-1}$. Recently, we prepared infusions $(100 \mathrm{ml}$ water plus $1.0 \mathrm{~g}$ tea leaves) of a range of commercially available tea samples from different countries of origin, and measured the aluminium concentration by graphite furnace atomic absorption spectroscopy. The mean concentration of aluminium in tea made from 12 brands was $3.9 \mathrm{mg} \mathrm{l}^{-1}$, with a range of $2.7-4.9 \mathrm{mg} \mathrm{l}^{-1}$, which is at most only one tenth of the reported levels.

Although our results should allay some of the unease generated by the above publications, there is still no information regarding the bioavailability of aluminium from different dietary sources. Because it is probable that the extent to which aluminium is absorbed from the intestinal tract depends on its chemical form, further research is required to assess the bioavailability of aluminium from tea, compared with other sources in the diet, and to determine the fate of the absorbed aluminium within the body.

SUSAN J. FAIRWEATHER-TAIT AFRC Institute of Food Research,

\section{Colney Lane,}

Norwich NR4 7UA, UK

Geoffrey R. Moore

S. E. JEMIL FATEMI

School of Chemical Sciences,

University of East Anglia,

Norwich NR4 7TJ, UK

1. Pearce. F. New Scient. 114 (1559), 28 (1987)

2. Kajda, P.K. B.N.F. Nutr. Bull. 11, 149-151 (1986)

Hodgkinson, N. Sunday Times p. 22 (29 February, 1987)

4. Coriat, A.M. \& Gillard, R.D. Nature 321, 570 (1986).

\section{Increase of $T_{\mathrm{c}}$ in $\mathrm{YBa}_{2} \mathrm{Cu}_{3} \mathrm{O}_{7-\delta}$} by exposure to nitrogen

SIR-Recently, Matthews et al. ${ }^{\prime}$ have reported an increase in the superconducting transition temperature $\left(T_{c}\right)$ of $\mathrm{YBa}_{2} \mathrm{Cu}_{3} \mathrm{O}_{7-\delta}$ by as much as $40 \mathrm{~K}$ when using nitrogen as a heat exchange medium. In view of the surprising results, and of the fact that this area is riddled with irreproducibilities, we have reinvestigated the effect of nitrogen on the $T_{c}$ of this ceramic superconductor. Although we also get a nominal increase in $T_{\mathrm{c}}$ by $\sim 20 \mathrm{~K}$ when measured in a nitrogen atmosphere, we feel that the increase is entirely due to a measurement artefact.

Our samples were made by the usual

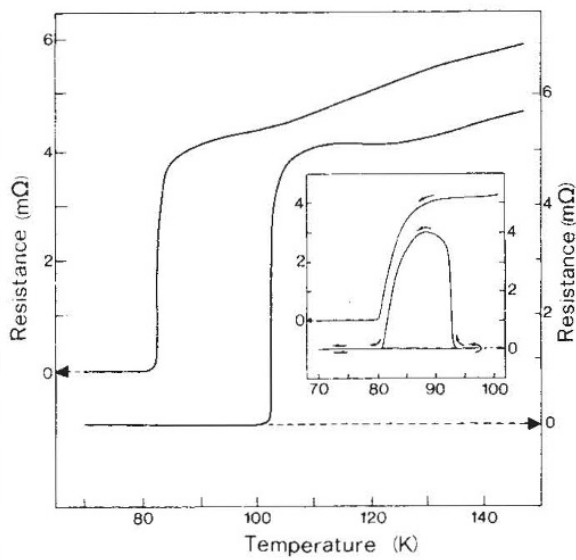

Fig. 1 Resistance versus temperature on cooling $(T=83 \mathrm{~K})$, and subsequent warming $\left(T_{\mathrm{c}}=103 \mathrm{~K}\right)$. The inset shows identical cooling behaviour, and what happened when we warmed the sample back to $100 \mathrm{~K}$ and then recooled it (as shown by the arrows).

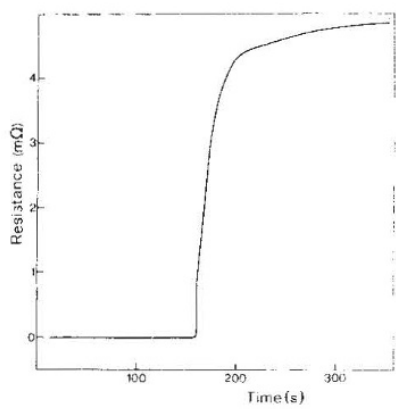

Fig. 2 Resistance versus time, while holding the temperature constant at $93 \mathrm{~K}$. The time at zero corresponds to $300 \mathrm{~s}$ after warm-up to $93 \mathrm{~K}$

ceramic method and the electrical resistivity was measured by the standard fourprobe technique. We chose a sample of $\mathrm{YBa}_{2} \mathrm{Cu}_{3} \mathrm{O}_{\gamma_{-\delta}}$ with $T_{\mathrm{c}}=83 \mathrm{~K}$, such that $T$ was about midway between the boiling points of $\mathrm{N}_{2}$ and $\mathrm{O}_{2}$. Figure 1 shows the resistance versus temperature for a typical cooling and heating cycle. During cooling, $T_{\text {c }}$ was always found to be $83 \mathrm{~K}$, whereas heating resulted in a $T_{c}$ of $103 \mathrm{~K}$. Our next measurement consisted of cooling the sample from 140 to $70 \mathrm{~K}$ (with $T_{\mathrm{c}}=83 \mathrm{~K}$ ), followed by warming the sample up to $100 \mathrm{~K}$, at which temperature it continued to be in the superconducting state (see Fig. 1 inset). When the sample was cooled again, we found a transition to the normal state at $T_{\mathrm{c}}=95 \mathrm{~K}$ and, surprisingly, a subsequent re-entry into the superconducting state at $83 \mathrm{~K}$ (Fig. 1 inset). This experiment was followed by cooling the sample to $70 \mathrm{~K}$, warming it up to $93 \mathrm{~K}$, where it was still superconducting, and holding the sample at constant temperature (within $\pm 1 \mathrm{~K}$ ) for several minutes. The resulting resistance versus time behaviour is shown in Fig. 2. This curve shows that the enhancement of $T_{\mathrm{c}}$ is temporary.

The above results are easily explained by assuming condensation of nitrogen in the sample when it is cooled to $77 \mathrm{~K}$, owing to the intrinisic porosity of the ceramic material used. Depending on the time spent above $77 \mathrm{~K}$, the stored liquid nitrogen keeps the sample below the superconducting transition temperature for some time, even when the environment temperature, monitored by a platinum resistor, is raised considerably above $T$. This leads to the artefact of an apparent increase in $T_{c}$. Although this mechanism certainly seems to be operative in our studies, one can only conjecture as to whether this artefact may have been responsible for the observations reported in ref. 1. But we find it significant that the enhancement of $T_{c}$ in ref. 1 was also observed on the warming cycle and only after condensation of nitrogen. This makes us suspect the same mechanism for the reported "enhancement" of $T_{c}$. Before concluding, we note that, in our experiments, the use of oxygen as an exchange 